\title{
Visual working memory for global, object, and part-based information
}

\author{
Michael D. Patterson \\ Nanyang Technological University, Singapore \\ BENJAMin MARTIN BLY \\ University of Medicine and Dentistry of New Jersey, Newark, New Jersey \\ ANTHONy J. Porcelli \\ Rutgers University, Newark, New Jersey \\ AND \\ BART RYPMA \\ University of Texas, Dallas, Texas \\ and University of Texas Southwestern Medical Center, Dallas, Texas
}

\begin{abstract}
We investigated visual working memory for novel objects and parts of novel objects. After a delay period, participants showed strikingly more accurate performance recognizing a single whole object than the parts of that object. This bias to remember whole objects, rather than parts, persisted even when the division between parts was clearly defined and the parts were disconnected from each other so that, in order to remember the single whole object, the participants needed to mentally combine the parts. In addition, the bias was confirmed when the parts were divided by color. These experiments indicated that holistic perceptual-grouping biases are automatically used to organize storage in visual working memory. In addition, our results suggested that the bias was impervious to top-down consciously directed control, because when task demands were manipulated through instruction and catch trials, the participants still recognized whole objects more quickly and more accurately than their parts. This bias persisted even when the whole objects were novel and the parts were familiar. We propose that visual working memory representations depend primarily on the global configural properties of whole objects, rather than part-based representations, even when the parts themselves can be clearly perceived as individual objects. This global configural bias beneficially reduces memory load on a capacity-limited system operating in a complex visual environment, because fewer distinct items must be remembered.
\end{abstract}

As we move through the world in daily life, we must remember the identity of objects and their locations in the visual environment. Working memory (WM) research has demonstrated that visual WM is limited to around three to four items (Duff \& Logie, 1999; Intraub, 1997; Kumar \& Jiang, 2005; Logie, 1995; Luck \& Vogel, 1997; Vogel, Woodman, \& Luck, 2001). In this article, we propose that visual memory for objects depends on the abstraction of incomplete, holistic representations constrained by the limited capacity of visual WM.

Researchers have posited two kinds of holistic representations in visual WM: global configural and objectbased representations. Global configural representations permit focus on overall structural properties, rather than on pieces of the visual environment. Focusing on such properties may allow compression of visual information in WM because exact details of scenes are not encoded. The use of object-based representations offers another possible method by which to reduce WM load. Focusing on objects, rather than on object parts, would limit memory load requirements, because not all parts of objects would need to be encoded distinctly. Object-based representations differ from global configural representations in that the representation is focused on only a limited area, rather than on the complete visual scene.

Results from several visual WM studies indicate that global configural information, such as the spatial location of visual features relative to one another, are well remembered. Santa (1977) found that participants encoded the spatial locations of three geometric shapes (e.g., a square, a triangle, and a circle) even when they were explicitly instructed to encode only the identity of the shapes, and not their locations. When the participants were tested on their recognition memory for shapes whose locations had been

B. Rypma, bart.rypma@utd.edu 
changed, accuracy decreased, as compared with when the locations remained the same. In another study, changing the spatial location of individual objects impeded short-term memory for colors and shapes, but changing the shape or color of the objects did not decrease shortterm memory for locations (Jiang, Olson, \& Chun, 2000). When the orientation of elongated lines of dots changed between the target and the probe, memory for the location of the dots decreased, as compared with when the direction of the elongated lines remained the same even when the participants were instructed to ignore the lines (Jiang, Chun, \& Olson, 2004). These studies suggest that use of global spatial configural information is obligatory. Using global configural visual properties, rather than focusing on each detail separately, may reduce overall visual WM load. Global configural information may also be used to organize information in visual WM, because when the structural properties of a scene are changed, memory for the scene decreases (Biederman, Glass, \& Stacy, 1973).

In addition to global configural properties, visual memory may also rely upon object-based information. Several studies have demonstrated that participants have a bias to focus on individual objects, both when using visual attention and in visually based recall. Visual attention studies indicate that attention tends to be focused on individual objects (Behrmann, Zemel, \& Mozer, 1998; Duncan, 1984). In one study, Duncan required participants to make tilt direction and texture-type judgments about two simultaneously displayed objects, a box and an intersecting line. The participants were more accurate and faster making two judgments about the same object than making one judgment each of different objects even when spatial proximity was controlled. In another study in which two objects consisting of overlapping rectangles were used, participants judged more quickly the similarity of bumps on two ends of the same object than that of bumps on the ends of two different objects, even when the bumps on the different objects were closer together than the bumps on the same object (Behrmann et al., 1998).

Change detection studies also indicate that participants are better at detecting changes on the object of focus than at detecting changes on another object even when the changes are equidistant from the point of focus (Woodman, Vecera, \& Luck, 2003). Change detection performance, where information is retained over very short delays, is thought to reflect visual WM (for a review, see Vogel et al., 2001). These results suggest the hypothesis that, as in visual attention tasks, object-based representations influence WM task performance. ${ }^{1}$

A central question is which grouping principle takes priority in visual WM. Duncan (1984) proposed that visual attention is organized hierarchically, so that attention may first be focused on objects and then parts of objects (see also Palmer, 1977; Vecera, Behrmann, \& Filapek, 2001). As has been reviewed above, a third level of grouping used in visual WM is global spatial configural grouping. Extending Duncan's hypothesis to visual WM, global spatial configural information may be at the top level of the hierarchy, followed by object-based information and then part-based information.
We conducted a series of experiments in order to examine the organization of visual WM by comparing memory for global configural information with object-based memory and part-based memory. Here, we define global configural information as information related to the overall structural form of the entire visual stimulus shown at encoding. We define an object as a limited area of the visual stimulus, defined by gestalt grouping principles (i.e., a series of contiguous line segments shown at encoding). We define a part as a subset of an object, which, in this series of experiments, can be considered a subset of contiguous line segments shown at encoding.

According to the hierarchical hypothesis, memory for global configural information should be most accurate, followed by memory for the individual objects and then for the parts of the objects. An alternate hypothesis is that consciously directed attention affects the representation of visual information in working memory. This hypothesis predicts that the level at which attention is focused will be recalled the most accurately. Thus, participants will remember parts or objects better than global configural information when they are instructed to focus on the individual parts or objects but will remember global configural information better when they are instructed to focus on the relation of each item to other items. We will refer to this hypothesis, as the attention-directed visual WM hypothesis. A third possibility is that global configural and object- and part-based representations may be given equal weight in visual WM, so that memory is equivalent for all three representations. There is, however, little empirical support for this notion.

Note that the task in this study was specifically designed to limit the participants to using only visual WM. The stimuli do not resemble visual objects already in long-term memory (LTM), and thus, participants should be generally unable to rely upon verbal WM or visual LTM. In addition, because individual stimuli do not repeat across trials, we provided little opportunity for learning specific visual stimuli.

\section{GENERAL METHOD}

In each experiment, separate groups of participants were shown the same stimuli but were instructed to focus memory on different levels of the stimuli. Experiments 1-4 tested the flexibility and bias of WM for two levels of representation. In the first experiment, we tested object- versus part-based memory. In Experiments 2 and 3, we tested object- versus global-configural-based memory. In Experiment 4, we used an alternate method to test object- versus globalconfigural-based memory. Finally, in Experiment 5, we compared memory for all three levels of representation. Each task consisted of three different periods: an encoding period, in which the visual stimulus was displayed; a maintenance period, in which the participants were instructed to hold the visual stimulus in memory; and a retrieval period, in which the participants were asked to compare the visual stimulus in memory with a probe.

\section{EXPERIMENT 1 Five-Line-Segment Objects}

In Experiment 1, the participants viewed objects consisting of five contiguously connected line segments dur- 
ing a 4-sec encoding period (see Figure 1). After an 8-sec maintenance period, they were given a 4-sec recognition test of their memory for the stimulus during the retrieval period. The whole-object group was instructed to keep the whole five-line-segment object in memory during the maintenance period. A whole object was defined as an object made up of all of the line segments shown at encoding. The part group was instructed to keep parts of the object in memory. Parts were defined as a subset of the line segments shown at encoding. Both groups of participants were tested at the retrieval period on their memory both for parts and for the original undivided whole object. The goal of the first experiment was to determine whether there is a bias to keep parts of an object together in working memory and whether this bias could be overcome by explicit instructions. An 8-sec maintenance period was chosen in order to ensure that iconic memory had completely faded, so that we could examine the remaining representation in visual WM (Sperling, 1967).

If the participants automatically encoded the contiguous five-line-segment item as a single object, memory should be better for the whole objects than for the parts of the objects in both groups. If the participants could consciously manipulate the structure of information stored in visual WM, the ability to detect changes in parts should be better than the ability to detect changes to the whole object in the part group. The parts consisted of one, two, or three line segments. The size of the part would allow us to examine how detailed memory for parts of the object was stored in visual WM; the smaller the part, the more detail the participant would be required to remember to perform the task. In general, we focused on accuracy data, although response time (RT) was also measured to confirm that there were no speed-accuracy trade-offs.

\section{Method}

Participants. Forty-six undergraduates were recruited from Rutgers University, Newark, introductory psychology courses. Twentysix students (ages, 18-36 years; mean age, 20.2; 20 of them female) were assigned to the part group, and 20 (ages, 18-35 years; mean age, $21.1 ; 12$ of them female) were assigned to the whole-object group. All the participants received course credit.

Procedure. Prior to the experiment, all participants signed Rutgers-IRB approved consent and were briefed on the purpose of the experiment. In the experiment proper, each trial consisted of three time periods. In the encoding period, the participants viewed an object made of five contiguous line segments (see Figure 1) for $4 \mathrm{sec}$. In the maintenance period of the trial, the participants viewed a blank screen for $8 \mathrm{sec}$, during which they had been instructed to "keep the object in memory." In the retrieval period of the trial, a probe item appeared, and the participants were given $4 \mathrm{sec}$ to respond.

On $80 \%$ of the trials faced by the part group, the probe consisted of one to three conjoined line segments. The participants' task in these trials was to determine whether the image was part of the image they had seen immediately previously. In other words, the participants were asked to judge whether the probe item was a part of the whole object they had viewed during the encoding period. Half of the parts were foil parts that did not come from the whole object seen at encoding. The foils were created by replacing one or more of the line segments with a line segment of a different length and angle.

For the whole object group, the first two trial periods were identical to those for the part group. In the retrieval period, however, the participants were shown a five-line-segment object and were instructed to determine whether the image was the same as the image they had seen immediately previously. In other words, they were instructed to judge whether that object was identical to the object shown during the encoding period.

The experimenter observed the performance of all the participants on a series of practice trials to confirm that the participants understood the task. If a participant did not reach a criterion score $(70 \%$ overall accuracy) on the practice trials or desired more practice, the participant repeated the practice trials.

The remaining $20 \%$ of the trials in both groups were catch trials. For the whole-object group, the probe was a one- to three-line-

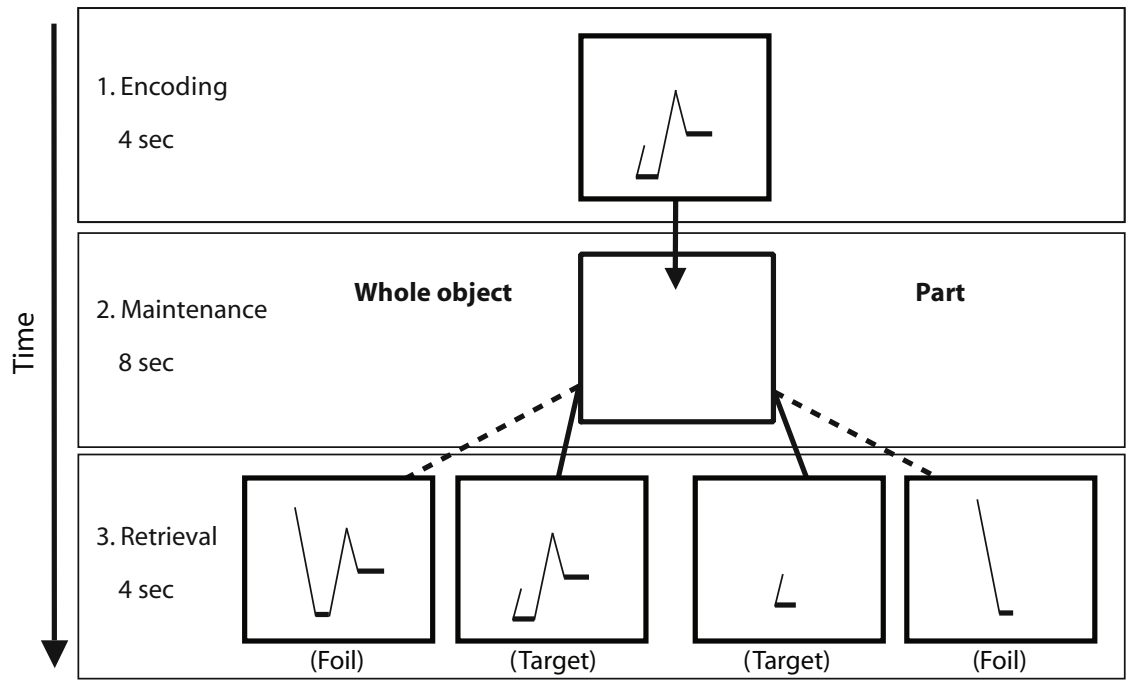

Figure 1. Example of the stimuli used in Experiment 1. The participants in both the wholeobject and the part groups saw the same five-line-segment stimulus during the 4-sec encoding period, followed by a blank screen that was presented for $8 \mathrm{sec}$. In the whole-object test condition, the participants were tested with a five-line-segment probe. In the part test condition, the participants were tested with a one-, two-, or three-line-segment probe. An example of a target (correct) and foil (incorrect) probe is given. 
segment part; for the part group, it was a five-line-segment object. The participants in the part group had been instructed that, on some trials, "instead of a part, you may be shown the same original image or a different image" and were shown five-line-segment examples; the participants in the whole-object group had been instructed that, on some trials, "you may be shown one of the parts of the original image" and were shown part stimuli examples. The experiment was executed using E-Prime software (Schneider, Eschman, \& Zuccolotto, 2002). The participants were instructed to press the "/" key for matching answers and " $Z$ " key for probe stimuli that did not match the stimuli shown during the encoding period.

\section{Results}

Accuracy and RT performance were measured. Accuracy was then converted into $A^{\prime}$ before statistical analyses were run (Pollack \& Norman, 1964). ${ }^{2}$ RT is reported for correctly answered trials only. Data from the part and the whole-object groups were analyzed together. An ANOVA using instructions (part or whole object) as a factor was calculated. Only data from match trials was included in the analyses. The participants were significantly more accurate in the whole-object group (.912) than in the part group (.687) $[F(1,142)=52.2, p<.00001]$. Analysis of RT data gave no indication of a speed-accuracy tradeoff, because a similar significant effect of instructions was found for RT, with the participants being slower judging parts $(2,032 \mathrm{msec})$ than judging whole objects $(1,756 \mathrm{msec})[F(1,142)=21.5, p<.0001]$.

An additional ANOVA using part size (one, two, or three line segment parts) as a factor was calculated for part group participants only. Accuracy, as measured by $A^{\prime}$, was significantly lower for one-line-segment parts than for two- or three-line-segment parts $[F(1,79)=25.938$, $p<.0001$; see Figure 2]. In the analysis of RT, there was not a significant effect of part size on RT $(F<1)$ and, thus, no indication of a speed-accuracy trade-off (see Table 1).

Catch trials. For the whole-object group, accuracy on match trials in $(86.1 \%)$ was greater than accuracy on catch trials $(79.4 \%)$, but the difference was not significant $[W=2,693, p=.5615$, Mann-Whitney test $]$. For the part group, accuracy for catch trials was significantly higher $(71.3 \%)$ than accuracy for match trials $(53.2 \%)$

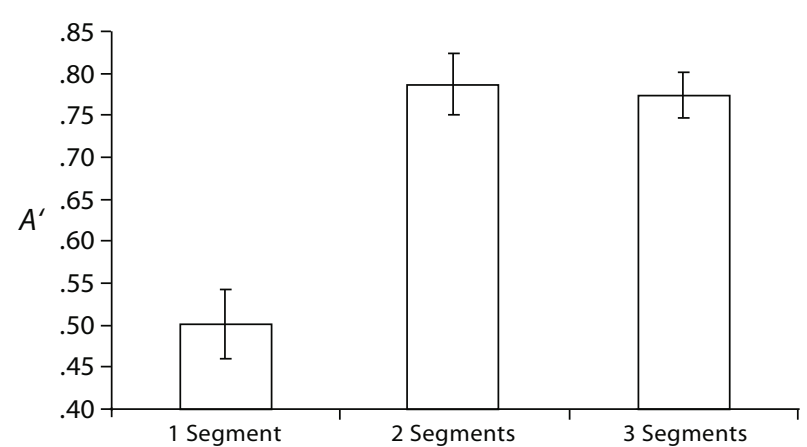

Figure 2. Effect of part size on accuracy in Experiment 1 for the participants in the part group. Accuracy for judging parts made of one line segment was significantly less than that for parts made up of two or three line segments.
Table 1

$A^{\prime}$ and Response Time (RT, in Milliseconds) Results From Experiment 1 in the Part Group

\begin{tabular}{lccccc}
\hline \multirow{2}{*}{ Probe } & \multicolumn{2}{c}{$A^{\prime}$} & & \multicolumn{2}{c}{ RT } \\
\cline { 2 - 3 } \cline { 5 - 6 } & $M$ & $S E$ & & $M$ & $S E$ \\
\hline Three lines & .774 & .027 & & 2,032 & 58.3 \\
Two lines & .786 & .037 & & 2,029 & 66.1 \\
One line & .501 & .041 & & 2,035 & 67.1 \\
\hline
\end{tabular}

$[W=1,973.5, p=.01927$, Mann-Whitney test $]$. These results indicated that the participants were not able to consciously alter strategy for this task, since in both instruction groups, accuracy was higher when a whole object was shown than when a part of the object was shown, although the differences were not significant.

\section{Discussion}

The results of Experiment 1 indicated that the participants had more difficulty recognizing parts of whole objects than they had recognizing whole objects. Furthermore, these results indicated that the participants were better able to recognize whole objects than parts even when they were instructed that they would be tested most frequently for parts. Although the part group participants' part performance was poor, they were well above chance in distinguishing between whole-object targets and foils, indicating a strong bias to keep contiguous lines together as one object in WM.

One possible alternative explanation of our results is that the participants had difficulty verifying the presence of part probe object parts because they had difficulty anticipating how to divide the object into parts. Another problem with the previous experiment is that, for the wholeobject group, no processing was required to transform the objects in WM, but for the part group, the participants first had to determine which part of the object was being tested and then compare that part with the object. Thus, judging object parts may have required more processing than judging whole objects and may have been inherently more difficult than remembering whole objects.

A third possible issue is that the participants in Experiment 1 appeared to process parts made of one line segment differently from parts made of two and three line segments. Figure 2 shows that accuracy was lowest for a part made of a single line segment. This result suggests that maintaining exact length and tilt information for individual line segments was more difficult than remembering a configuration of several line segments.

\section{EXPERIMENT 2}

\section{0-Line-Segment Shape Without Inner Lines}

In Experiment 2, we replicated the results of Experiment 1 and addressed the three alternative accounts outlined above. In this experiment, each whole object consisted of a total of 10 line segments that were divided into two, three, and four parts (see Figure 3). The outline of the whole object was clearly defined, so that the participants could determine the shape of a single whole object, and the 


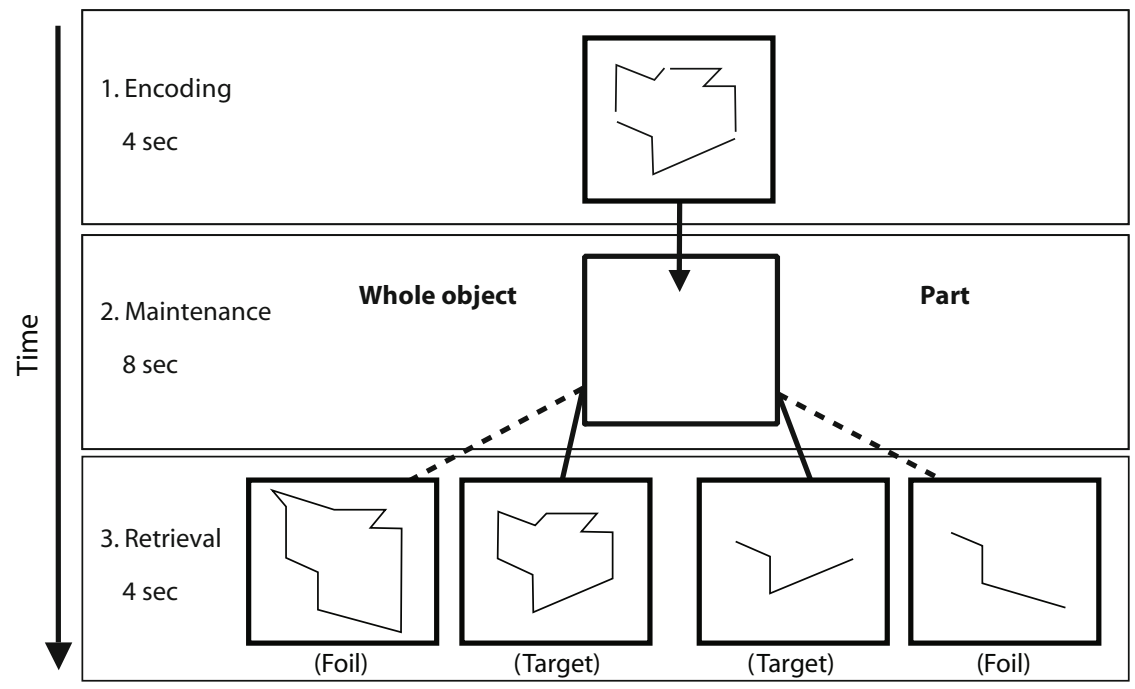

Figure 3. Example of the stimuli used in Experiment 2. The participants in both the wholeobject and the part groups saw the same 10-line-segment stimulus during the 4-sec encoding period, which was broken into two, three, or four parts, followed by a blank screen which was presented for $8 \mathrm{sec}$. In the whole-object test condition, the participants were tested with a 10-line-segment enclosed stimulus. In the part test condition, the participants were tested with one of the parts shown during encoding. An example of a target (correct) and foil (incorrect) probe is given.

division of each part was marked by a gap, so that the participants could also clearly determine the composition of each part. Second, in contrast to Experiment 1, the stimuli were designed so that remembering whole objects would require more processing than would remembering parts.

No transformation was necessary in the part condition, but in the whole-object condition, the participants had to imagine the parts moved together across the gaps so that the lines were joined to form the unbroken outline of the shape. Furthermore, more manipulation became necessary as the number of parts increased. Thus, it should have been more difficult to combine four parts into one whole object than to combine two parts into a whole object, because there were more gaps between parts as the number of parts increased. Assuming that the participants were successful at combining the parts into whole objects, WM load for each whole object should have been equivalent, no matter how many parts it consisted of originally. Thus, in the whole-object group, if the participants stored each stimulus as a single, holistic 10-line-segment object, we would expect accuracy and RT to be equivalent no matter how many parts each stimulus consisted of. However, for the part group, if the participants stored each part separately, we would expect RT to increase and accuracy to decrease as the number of parts increased. In Experiment 2 , none of the parts were made up of fewer than 2 line segments, in order to ameliorate the difficulty of remembering angles and lengths of single line segments that we found in Experiment 1.

An important question addressed in these experiments was that of whether or not attention could be used to improve WM for the several individual objects, instead of WM for global configural properties of whole objects.
Because each of the parts of the whole objects in Experiment 2 were separated by gaps, each part could be considered a separate individual object. In this experiment, we examined whether the participants would be capable of storing each part separately in memory. If the participants in the part group encoded each part separately, we would expect that accuracy would decrease and RT would increase as the number of parts to remember increased, because WM load would be closer to capacity. This result would indicate that the global configural bias in visual WM can be modified by top-down control.

\section{Method}

Participants. Twenty-two participants were recruited from Rutgers University, Newark, introductory psychology courses. Half of them (ages, 18-40 years; mean age, 20.6; 9 of them female) participated in the part group, and half (ages, 18-23 years; mean age, $19.2 ; 5$ of them female) participated in whole-object group. All the participants received course credit for participation.

Procedure. The same three periods and timing as those in the previous experiment were used. In this experiment, the participants were shown two, three, or four parts, which consisted of a total of 10 line segments at the beginning of each trial (see Figure 3). In the part group, the participants were asked to remember the individual parts of each object, and $80 \%$ of the time, they were shown a part consisting of 2-5 line segments. The task was to judge whether or not the part was one of the parts shown during the encoding period. In the whole-object group, the participants saw stimuli identical to those seen by the part group during the encoding period, but then, $80 \%$ of the time, they saw a whole object made up of a 10-line-segment enclosed shape. The task was to indicate whether that shape was the same as the shape that the parts displayed during the encoding period would form if they were joined together. Twenty percent of the time, the participants saw catch trials. In catch trials for the part group, the participants saw 10-line-segment enclosed shapes and were required to judge whether the enclosed shape could be formed if the line 


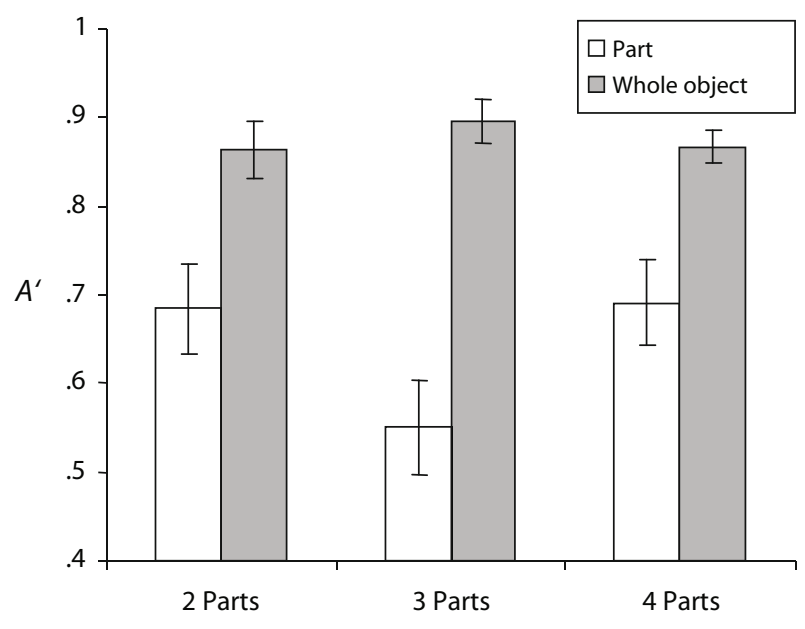

Figure 4. Effect of instructions and number of parts on accuracy in Experiment 2. Accuracy for whole objects was significantly higher than accuracy for parts. There was not a significant effect of number of parts shown during the encoding period.

segments seen at encoding were joined together. In the catch trials for the whole-object group, the participants saw one of the two- to five-line-segment parts and were required to judge whether that part was one of the parts shown at encoding.

\section{Results}

A 2 (part or whole-object instruction) $\times 3$ (two, three, or four parts) ANOVA was calculated on accuracy and RT, only for trials in the match condition. Accuracy scores were calculated as $A^{\prime}$, and RT was calculated only for correctly answered trials. A main effect of instruction was found on accuracy $[F(1,62)=45.95, p<.0001$; see Figure 4]. No other significant effects on accuracy were detected $(F \mathrm{~s}<1)$. Analysis of the RT data gave no indication of a speed-accuracy trade-off. RT was significantly longer in the part group $(2,051 \mathrm{msec})$ than in the whole-object group $(1,689 \mathrm{msec})[F(1,62)=11.63, p<.01$; see Table 2].

Catch trials. Catch trial $(76.7 \%)$ responses were significantly more accurate than those for match trials $(59.8 \%)$ in the part group $[W=283.5, p=.0007601$, Mann-Whitney test], but match trial $(80.4 \%)$ responses were significantly more accurate than those for catch trials $(54.6 \%)$ in the whole-object instruction group $[W=969.5, p<.0001$, Mann-Whitney test]. Thus, the participants judged whole objects more accurately than parts, even when they were instructed to focus on remembering parts.

\section{Discussion}

This experiment replicated the results from Experiment 1 , indicating a strong bias to remember a whole object in visual WM, rather than parts, even when the parts themselves could be considered distinct objects (see Figure 4). The bias was unaffected by top-down processing, because accuracy for parts was near chance in both groups but well above chance for whole objects, indicating that the participants were unable to change WM representations from global configural forms to nonglobal objectbased forms, despite instructions to do so.
In neither instruction group did we find load effects. This may indicate that in the whole-object group, the participants successfully manipulated the parts in WM so that they formed a single enclosed whole object. The lack of a load effect in the part group indicates an alternate possibility that the participants had difficulty perceiving each part separately because the gestalt properties of the outline of the whole object may have overridden the perception of individual sets of conjoined line segments. ${ }^{3}$ Another possible explanation for the results in Experiments 1 and 2 is that accuracy was better for whole objects because they were more similar to the stimulus shown at encoding than were the parts. Whole objects contained all the detail of the stimulus shown at encoding, but parts contained only a limited area of the stimulus. To eliminate these explanations, we performed Experiment 3.

\section{EXPERIMENT 3 10-Line-Segment Shape With Inner Lines}

In Experiment 3, stimuli were devised so that combining the parts to form a whole object would lead to a loss of detail from stimulus shown during the encoding period because, when the participants mentally combined the parts, only the outer outline of the parts would be preserved, and the inner lines of the parts would be subsumed into the whole object. The stimuli were similar to those in Experiment 2 , with the exception that inner lines caused each part to be an enclosed shape consisting of 7-10 line segments (see Figure 5). The outer lines of the parts consisted of a total of 10 line segments, and joining these outer line segments together produced a whole object. Unlike in the first two experiments, the whole object no longer contained many of the global spatial configural details.

Thus, this experiment allowed us to address the question of whether all global configural details are necessary for there to be a whole-object memory advantage. Because the whole object now consisted of 10 line segments and each part consisted of 7-10 line segments, both the whole-object and the part probes preserved approximately equivalent numbers of line segments from the original stimulus shown during the encoding period. Thus, we could test the hypothesis that accuracy was better in the whole-object groups in the previous two experiments because the whole-object probes included more detail in common with the stimulus presented at encoding than did the part probes.

Table 2

$A^{\prime}$ and Response Time (RT, in Milliseconds) Results From Experiment 2 in Whole-Object and Part Groups

\begin{tabular}{|c|c|c|c|c|c|c|c|c|}
\hline \multirow[b]{3}{*}{ No. of Parts } & \multicolumn{4}{|c|}{ Whole-Object Group } & \multicolumn{4}{|c|}{ Part Group } \\
\hline & \multicolumn{2}{|c|}{$A^{\prime}$} & \multicolumn{2}{|c|}{$\mathrm{RT}$} & \multicolumn{2}{|c|}{$A^{\prime}$} & \multicolumn{2}{|c|}{ RT } \\
\hline & $M$ & $S E$ & $M$ & $S E$ & $M$ & $S E$ & $M$ & $S E$ \\
\hline Two & .863 & .032 & 1,670 & 142 & 684 & .050 & 1,910 & 81.5 \\
\hline Three & .896 & .024 & 1,706 & 144 & .550 & .053 & 2,087 & 112 \\
\hline Four & .866 & .019 & 1,691 & 132 & 691 & .048 & 2,117 & 139 \\
\hline
\end{tabular}




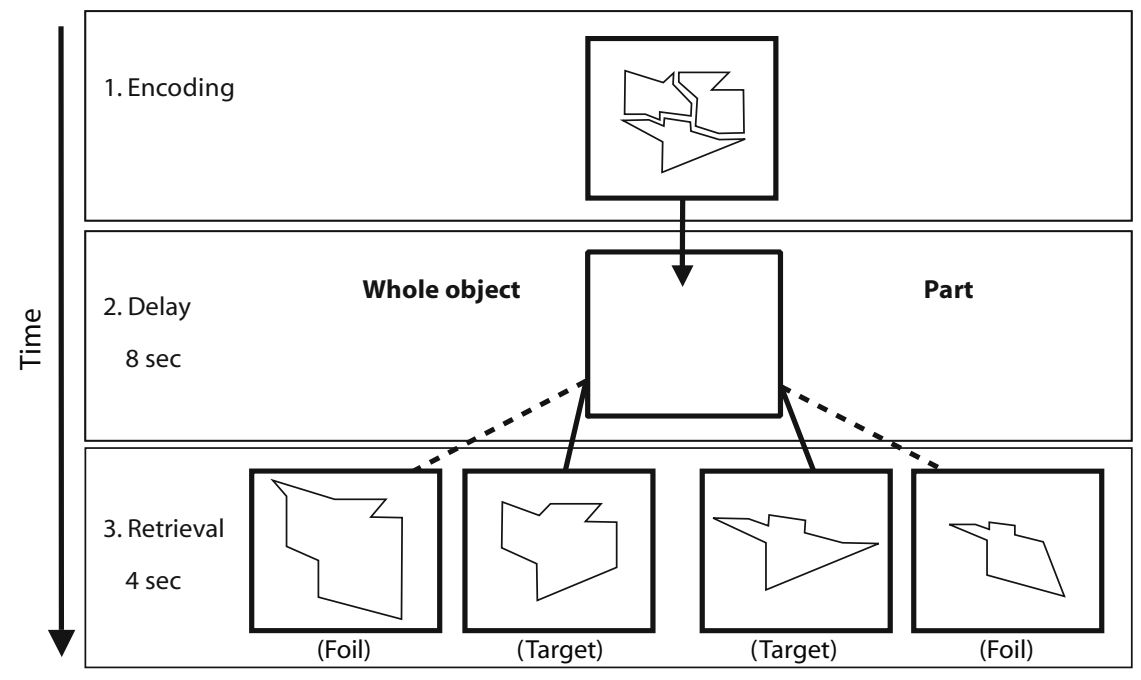

Figure 5. Example of the stimuli used in Experiment 3. The participants in both the wholeobject and the part groups saw the same stimulus during the 4-sec encoding period, which was broken into two, three, or four enclosed parts (each made up of $\sim 10$ line segments), followed by a blank screen that was presented for 8 sec. In the whole-object test condition, the participants were tested with a 10-line-segment enclosed probe. In the enclosed probe, only the outer lines of the encoded stimulus remained. In the part test condition, the participants were tested with one of the parts shown at the beginning of the trial. An example of a target (correct) and a foil (incorrect) probe is given.

Alternatively, retaining only the outline of the parts in order to remember the whole object may be advantageous because it makes smaller demands on visual WM. For example, to remember four 10-line-segment parts requires memory for a total of 40 line segments, but to remember one whole object made up of four parts requires memory for only 10 line segments. If this were the case, the memory advantage for whole objects would become stronger as the number of parts increased.

In addition, the division between the parts was more clearly marked in Experiment 3 than in Experiment 2. Thus, this experiment allowed us to address the possible explanation that there was a global configural bias in Experiments 1 and 2 because the participants had difficulty perceiving how the whole objects were divided into parts.

\section{Method}

Participants. Twenty-four participants were recruited from Rutgers University, Newark, introductory psychology courses. Half of them (ages, 18-21 years; mean age, 19.0; 9 of them female) participated in the part group, and half of them (ages, 18-42 years; mean age, $22.8 ; 9$ of them female) participated in the whole-object group. All the participants received course credit.

Procedure. The procedure was the same as that used in Experiment 2. The same stimuli were used, except that each part had inner lines, so that each part consisted of completely enclosed polygons made up of 7-10 line segments (see Figure 5).

\section{Results}

A 2 (whole or part instruction) $\times 3$ (two, three, or four parts) ANOVA was calculated on accuracy converted to $A^{\prime}$ and RT for correctly answered trials in the match condition. There was a significant main effect of instruc- tion on accuracy $[F(1,68)=8.47, p<.01$; see Figure 6]. There were no other significant effects on accuracy $\left(F_{\mathrm{s}}<2.5\right)$. Analysis of the RT data gave no indication of a speed-accuracy trade-off. RT for judging whole objects $(1,817 \mathrm{msec})$ was significantly shorter than that for judging parts $(2,340 \mathrm{msec})[F(1,68)=9.62, p<.01$; see Table 3].

Catch trials. The participants who received part instructions showed a trend of higher accuracy in the catch trials $(68.3 \%)$ than in the match trials $(61.3 \%)[W=485.5$, $p=.06605$, Mann-Whitney test], but those who received

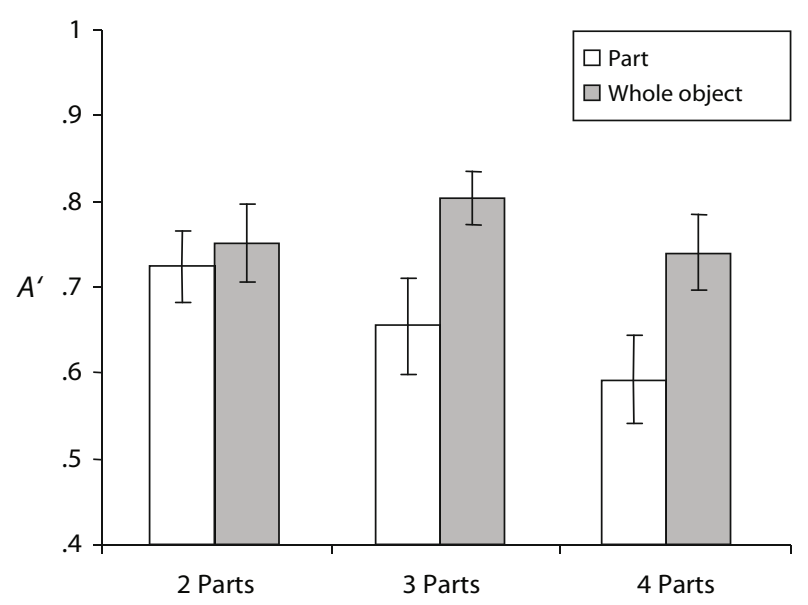

Figure 6. Effect of instructions on accuracy in Experiment 3. Accuracy for whole objects was significantly higher than accuracy for parts. There was not a significant effect of number of parts shown during the encoding period. 
Table 3

$A^{\prime}$ and Response Time (RT, in Milliseconds) Results From Experiment 3 in Whole-Object and Part Groups

\begin{tabular}{|c|c|c|c|c|c|c|c|c|}
\hline \multirow[b]{3}{*}{ No. of Parts } & \multicolumn{4}{|c|}{ Whole-Object Group } & \multicolumn{4}{|c|}{ Part Group } \\
\hline & \multicolumn{2}{|c|}{$A^{\prime}$} & \multicolumn{2}{|c|}{ RT } & \multicolumn{2}{|c|}{$A^{\prime}$} & \multicolumn{2}{|c|}{ RT } \\
\hline & $M$ & $S E$ & $M$ & $S E$ & $M$ & $S E$ & $M$ & $S E$ \\
\hline Two & .762 & .048 & 1,942 & 109 & .735 & .043 & 2,243 & 122 \\
\hline Three & .817 & .033 & 1,909 & 114 & .663 & .058 & 2,250 & 116 \\
\hline Four & .751 & .046 & 1,758 & 105 & .598 & .053 & 2,200 & 127 \\
\hline
\end{tabular}

whole-object instructions were significantly more accurate on match trials $(70.8 \%)$ than on catch $(53.2 \%)$ trials $[W=983, p=.0001479$, Mann-Whitney test $]$. These results indicated that although instructions did have some effect on accuracy, they did not alter the bias to retain the stimulus' global configural properties.

\section{Discussion}

As in the first two experiments, the participants more accurately remembered whole objects than parts. Even in the part group, accuracy was better for a whole object than for parts, indicating that the participants automatically grouped the items into a composite consisting of the outline of the stimuli, even though they were instructed to remember each part individually. Furthermore, the number of line segments that were present in the probe of the whole-object group was roughly equivalent to the number of line segments present in the probe of the part group. These results do not support the hypothesis that accuracy was better in the whole-object group simply because it included more detail in common with the stimulus presented at the beginning of the trial. They support the hypothesis of a bias to encode the overall shape of the stimuli in WM, rather than individual details.

Accuracy was lower in Experiment 3 than in Experiment 2 for judging whole objects. There are two possible reasons for the accuracy decrease. First, there were more total lines to remember in Experiment 3 than in Experiment 2. Second, in Experiment 3, only the outer lines of the original visual stimulus were preserved in the probe. Nevertheless, there was still a WM advantage for the whole object, as compared with the part group, indicating a bias to remember the overall outline of a visual stimulus even when the inner lines form the boundaries of polygons. Further research is needed to determine whether outer outlines of shapes have a privileged status in WM. One experiment that might resolve this issue would involve testing WM only for inner line segments of parts joined together.

\section{EXPERIMENT 4}

Experiments 1-3 demonstrated that there was a bias in visual WM for the global configural properties of visual stimuli, even when the parts were individual objects themselves. Experiment 4 tested the generalizability of the global configural WM bias. In Experiments 2 and 3, the parts were divided from each other by gaps. One pos- sibility is that the bias for global configural properties occurs only when parts are subdivided by gaps. In Experiment 4 , parts were defined as lines that were of the same color.

Another possible explanation for the global configural bias is that the method used to divide the whole objects into parts produced parts that were particularly difficult to process. Specifically, previous research has shown worse memory for parts each consisting of several disconnected line segments than for parts consisting of connected line segments (Carlson-Radvansky \& Irwin, 1995; Palmer, 1977; Palmer \& Rock, 1994). Thus, in Experiment 4, we tested the generalizability of the results by dividing parts, using two different methods. Good parts consisted of contiguous line segments and were similar to the parts used in Experiments 1-3 (see Figure 7A). Bad parts consisted of noncontiguous line segments (see Figure 7B).

Third, in Experiment 4, catch and match trials were displayed with equal frequency, allowing us to statistically compare the effects of instruction and probe type separately. In the previous three experiments, we analyzed only trials on which the instructions and the probe type matched, because catch trials were displayed only $20 \%$ of the time and, thus, not enough data were collected to be submitted to a full analysis.

One difference between the part and whole object conditions in the previous experiments was that, for the whole-object group, the part group faced more variability in adapting to encoding either two, three, or four parts per trial, whereas the whole-object group did not need to switch strategies each trial, encoding only one whole object per trial. Adapting more flexible strategies may require more central executive processing (Rypma, 2006; Rypma, Berger, \& D'Esposito, 2002). In Experiment 4, there were always three parts per trial, so that the participants in the part group did not alter strategies each trial and, thus, executive demands between the whole-object and the part groups were similar.

\section{Method}

Participants. Twenty-four participants were recruited from Rutgers University, Newark, introductory psychology courses. Half of them (ages, 19-34 years; mean age, 23.1; 7 of them female) participated in the part group, and the other half (ages, 18-26 years; mean age, 19.1; 9 of them female) participated in the whole-object group. All the participants received course credit.

Procedure. The same procedure as that in Experiment 2 and 3 was used, with the exception that after each trial, the participants were given feedback. Each stimulus consisted of a total of 10 line segments that were connected, with no gaps to form a 10-linesegment polygon. The 10 line segments were divided into either three bad parts or three good parts (see Figure 7). Each part was defined by a color: black, red, or blue. In the good part condition, each part consisted of 3-4 conjoined line segments. In the bad part condition, each part consisted of 3-4 line segments, with no consecutive line segments that were the same color. The participants were instructed that there were three parts shown at the beginning of each trial, with each part defined by a color. The part group was instructed to remember "each part separately," and the whole-object group was instructed to remember the "whole shape" shown at the encoding period.

The stimuli and probes shown to the part and the whole-object instruction groups were identical. During half of the encoding pe- 
A

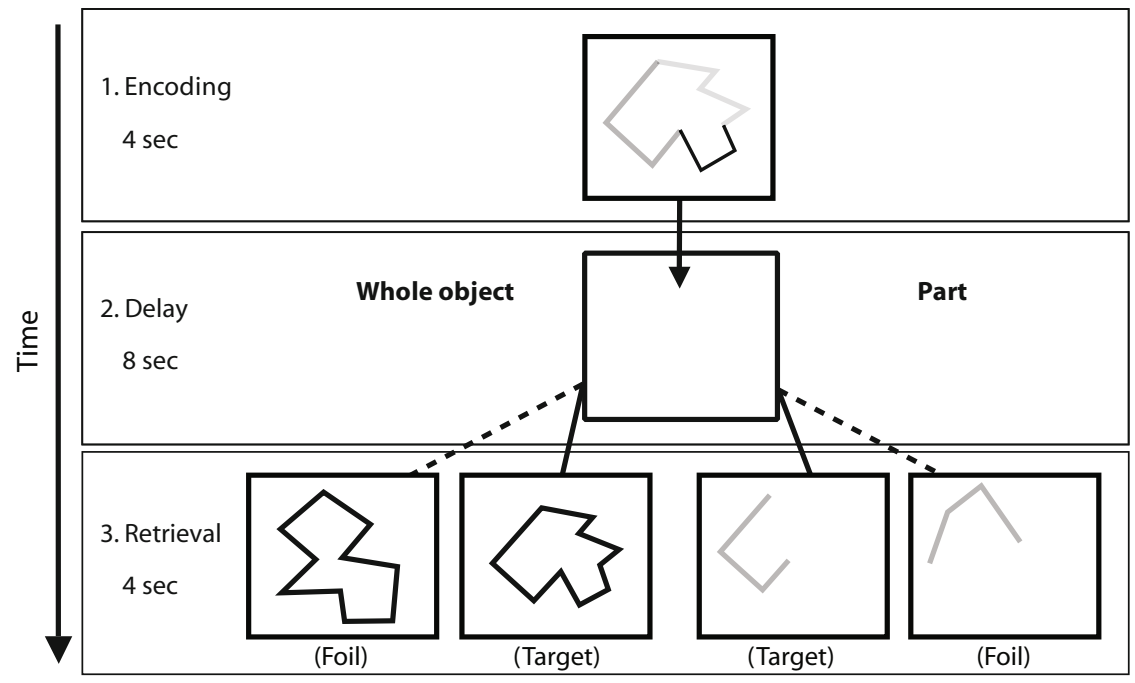

B

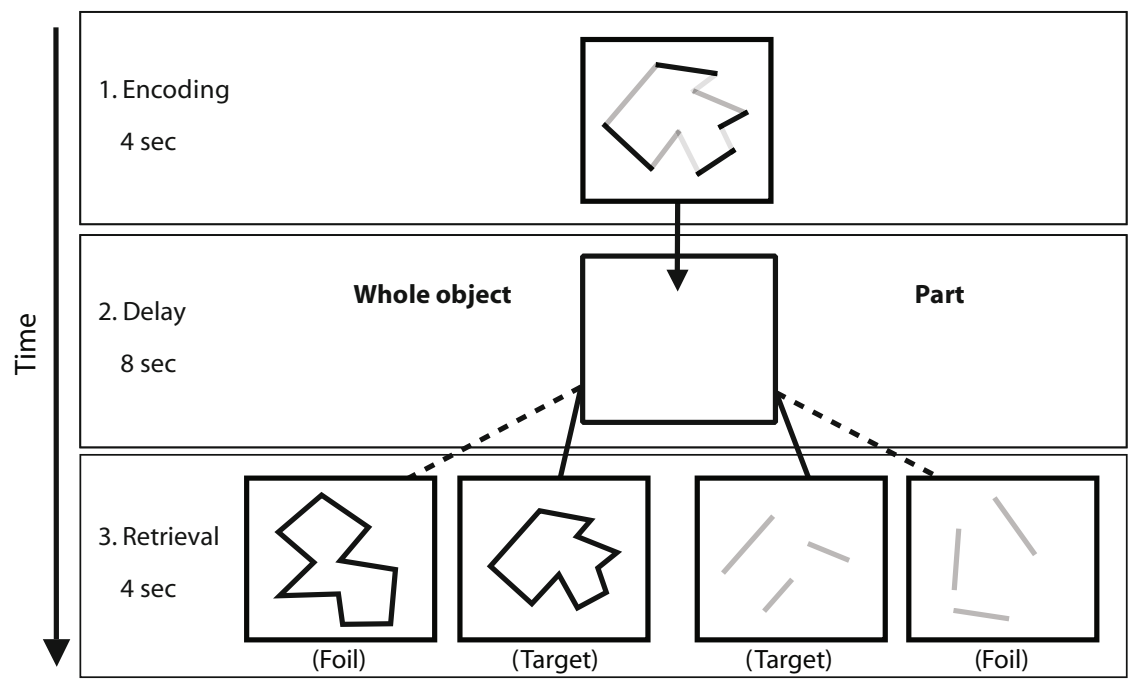

Figure 7. (A) Stimuli for good parts. (B) Stimuli for bad parts. The participants in both the whole-object and the part groups saw the same stimulus during the 4-sec encoding period, which was broken into three colored parts, followed by a blank screen that was presented for $8 \mathrm{sec}$. In the experiment, the lines were colored brown (solid black lines), red (dark gray lines), and blue (light gray lines). In the whole-object test condition, the participants were tested with a black 10-line-segment enclosed probe. In the part group, the participants were tested with one of the colored parts shown at the beginning of the trial. An example of a target (correct) and a foil (incorrect) probe is given.

riods, whole objects with colored lines delineating good parts were shown, and during the other half, whole objects with colored lines delineating bad parts were shown. During the retrieval period, the participants were shown either a part or a whole object. The whole object was made up of only black line segments. Fifty percent of the time, the shape of the whole object was the same as the shape displayed at the beginning of the trial, and $50 \%$ of the time, a different shape was displayed. In the part probe condition, a part was displayed that was made up of red, blue, or black line segments. The type of part probe shown during the retrieval period matched the type of stimulus shown during the encoding period. For trials in which good parts were displayed during the encoding period, good parts (consisting of connected line segments) were displayed during the retrieval period; when bad parts (consisting of disconnected line segments) were shown at the encoding period, bad parts were displayed during the retrieval period. Fifty percent of the time, the part probe matched a part displayed during the encoding period.

\section{Results}

A 2 (whole or part instruction) $\times 2$ (bad or good part type) $\times 2$ (whole or part) ANOVA was calculated on accuracy and RT data. Note that match and catch trials were included in the same analysis. Accuracy was converted to $A^{\prime}$ before analysis, and only correct trials were included 


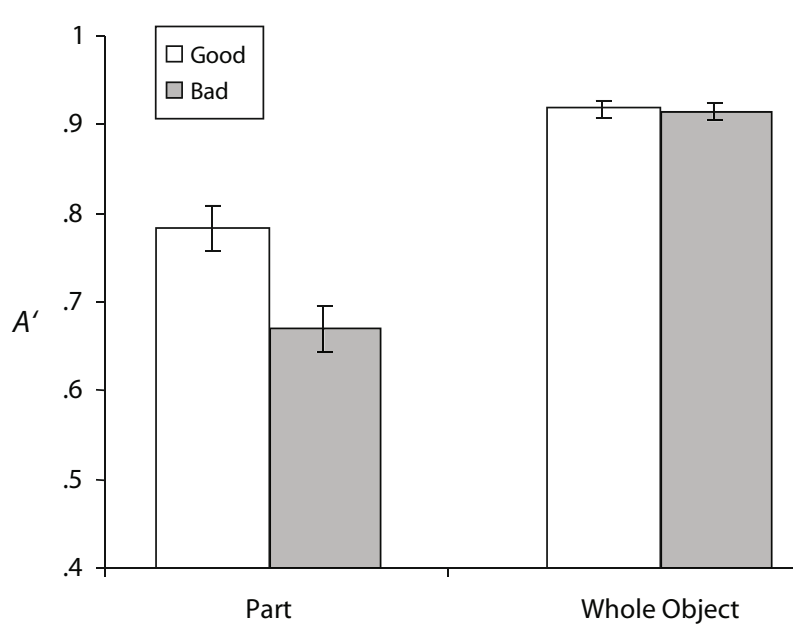

Figure 8. Effect of part type and test condition on accuracy in Experiment 4. Accuracy was significantly higher for whole objects than for parts. Accuracy was significantly higher for good parts than for bad parts.

in the RT analysis. There was a significant main effect of probe type on accuracy $[F(1,88)=87.9, p<.0001]$. There was also a significant main effect of part type on accuracy $[F(1,88)=8.09, p<.01]$ and a significant interaction between probe type and part type $[F(3,88)=$ 7.65, $p<.01$; see Figure 8]. Analysis of RT data gave no indication of a speed-accuracy trade-off. The only significant effect on RT was that whole probes $(1,084 \mathrm{msec})$ were judged more quickly than part probes $(1,324 \mathrm{msec})$ $[F(1,88)=13.3, p<.001$; see Tables $4 \mathrm{~A}$ and $4 \mathrm{~B}]$.

\section{Discussion}

Experiment 4 replicated the results from the previous experiments indicating a bias in visual WM for global configural information. In Experiment 4, the effect of instructions and probe type were examined individually. Instructions had no effect, since the participants were better at judging whole-object probes independently of the type of instructions they had received. This result suggests that the global configural bias is not affected by top-down control.

Accuracy was equivalent for judging whole objects when the stimuli were divided into bad or good parts. These results suggested that the participants did not pay attention to the composition of parts when encoding visual stimuli. However, the participants had more difficulty deciding whether a bad part seen at encoding matched a bad part seen at retrieval than deciding whether a good part seen at encoding matched a good part seen at retrieval. This result indicated that the parts used in the first three experiments were not especially difficult, as compared with how the bad parts were divided. Further research is needed to determine what parameters distinguish good and bad parts. Nonetheless, these results support the hypothesis that the visual WM system is biased toward retention of whole objects, rather than parts, no matter how the parts are constructed.
Furthermore, these results demonstrated that previous results were not due to higher demands for flexibility of processing in the part group-since in Experiment 4, the number of parts shown in each trial was constant-and more clearly demonstrated that the participants were unable to consciously change the bias to remember global configural details better than lower level part- or objectbased details. Note that whole-object probes were black and, thus, differed from the colored stimuli shown during the encoding period but that the part probes used the same colors as those shown during the encoding period. Thus, even though the whole-object probes differed more from the encoding period stimuli than did the part probes, the participants were substantially better at correctly remembering whole objects than at remembering parts.

In the previous experiments in this study, only two levels of visual processing were tested during each experiment. This feature limited our ability to test the proposed hierarchical representation hypothesis because, so far, none of the experiments had permitted simultaneous comparison of all three levels (i.e., global configural, object based, and part based). Experiment 5 addressed this problem.

\section{EXPERIMENT 5}

There are two kinds of holistic visual processing that can be used to reduce the number of items stored in visual WM. Global configural processing reduces the entire visual scene to a single representation. Object-based processing represents limited areas of the scene defined by gestalt grouping properties. Beneath these two levels of processing, part-based processing represents limited sections of an object. According to the proposed hierarchical representation hypothesis of visual WM, visual WM is best for global configural details, followed by object-based and then part-based details. Although the hierarchical representation hypothesis is based on three levels, only two levels have been tested in each of the prior Experiments

Table 4A

$A^{\prime}$ and Response Time (RT, in Milliseconds) Results From Experiment 4 in Whole-Object and Part Instruction Groups Using Good Parts

\begin{tabular}{|c|c|c|c|c|c|c|c|c|}
\hline \multirow[b]{3}{*}{ Type of Probe } & \multicolumn{4}{|c|}{ Whole-Object Group } & \multicolumn{4}{|c|}{ Part Group } \\
\hline & \multicolumn{2}{|c|}{$A^{\prime}$} & \multicolumn{2}{|c|}{ RT } & \multicolumn{2}{|c|}{$A^{\prime}$} & \multicolumn{2}{|c|}{ RT } \\
\hline & $M$ & $S E$ & $M$ & $S E$ & $M$ & $S E$ & $M$ & $S E$ \\
\hline Whole & .918 & .017 & 1,140 & 55.8 & .918 & .014 & 1,100 & 110 \\
\hline Part & .797 & .036 & 1,312 & 72.2 & .770 & .040 & 1,321 & 125 \\
\hline
\end{tabular}

Table 4B

$A^{\prime}$ and Response Time (RT, in Milliseconds) Results From Experiment 4 in Whole-Object and Part Instruction Groups Using Bad Parts

\begin{tabular}{|c|c|c|c|c|c|c|c|c|}
\hline \multirow[b]{3}{*}{ Type of Probe } & \multicolumn{4}{|c|}{ Whole Instructions } & \multicolumn{4}{|c|}{ Part Instructions } \\
\hline & \multicolumn{2}{|c|}{$A^{\prime}$} & \multicolumn{2}{|c|}{ RT } & \multicolumn{2}{|c|}{$A^{\prime}$} & \multicolumn{2}{|c|}{ RT } \\
\hline & $M$ & $S E$ & $M$ & $S E$ & $M$ & $S E$ & $M$ & $S E$ \\
\hline Whole & .916 & .012 & 1,070 & 50.1 & .916 & .017 & 1,024 & 92.0 \\
\hline Part & .648 & .044 & 1,376 & 94.1 & 692 & .029 & 1,288 & 117 \\
\hline
\end{tabular}


1-4. Thus, in Experiment 5, we compared all three levels at once: global configural information, defined as the entire visual stimuli; object-based information, defined by conjoined line segments; and part-based information, defined by lines of the same color (see Figure 9). We used three groups of participants, all of whom were shown the same stimuli but were given instructions to focus on the global configural, object-based, or part-based information. If, as we claim, global configural information has privileged status in visual WM, there should be minimal effect of instructions on performance.

\section{Method}

Participants. Twenty-nine participants were recruited from Rutgers University, Newark, introductory psychology courses and received course credit for participation. Seventeen participants (ages, 18-24 years; mean age, $19.7 ; 9$ of them female) were assigned to the part group. A second group of 12 participants (ages, 18-22 years; mean age, $19.4 ; 9$ of them female) were assigned to the object group. Finally, 12 participants (ages, 18-51 years; mean age, 23.5; 10 of them female) were assigned to the whole group. All the participants received course credit.

Procedure. The same procedure as that in Experiment 4 was used. Each stimulus consisted of a total of 10 line segments. The 10 line segments were divided into two objects. Each object was made up of 5 connected line segments. The two objects were separated by gaps. Each object was made up of two parts, each consisting of 2-3 connected line segments. Each of the four parts was displayed in a different color: red, blue, green, or brown. The participants were instructed that there were three kinds of representations present in the stimulus at each encoding period: the whole shape, consisting of 10 lines; two objects, each consisting of 5 conjoined line segments; and four parts, each consisting of connected lines made up of a single color (see Figure 9). The participants in the whole-object group were instructed to focus on remembering the whole shape, the partici- pants in the object group were instructed to focus on remembering each object made up of conjoined lines, and the participants in the part group were instructed to focus on remembering parts made up of the same color.

As in Experiment 4, to separate the effect of instruction and probe condition, the participants in all three instruction groups were tested equally at each level: part, object, or global configural details (each one third of the trials). The global configural test consisted of a shape made up of 10 conjoined black line segments. Fifty percent of the time, the shape matched the shape shown during the encoding period (with the exception that no gaps existed in the probe shape). The object group was shown five conjoined black lines. Fifty percent of the time, the 5 line segment object matched an object that was shown at encoding. Finally, in the part probe condition, the participants were shown a black part made up of 2-3 conjoined line segments. Fifty percent of the time, it matched the shape of one of the parts shown at encoding.

\section{Results}

A 3 (whole, object, or part instructions) $\times 3$ (whole, object, or part probe type) ANOVA was calculated on accuracy converted to $A^{\prime}$ and RT for correct answers only. The only significant effect was a main effect of probe type on accuracy $[F(2,114)=29.9, p<.0001$; see Table 5]. Post hoc $t$ tests, corrected for multiple comparisons, indicated that all the conditions were significantly different from each other (see Figure 10). There were no significant effects of instructions, and there were no significant RT effects, confirming that there was no speed-accuracy trade-off. The mean RT was $1,190 \mathrm{msec}$.

\section{Discussion}

Experiment 5 replicated the previous result that the participants recalled the global configural detail of a whole

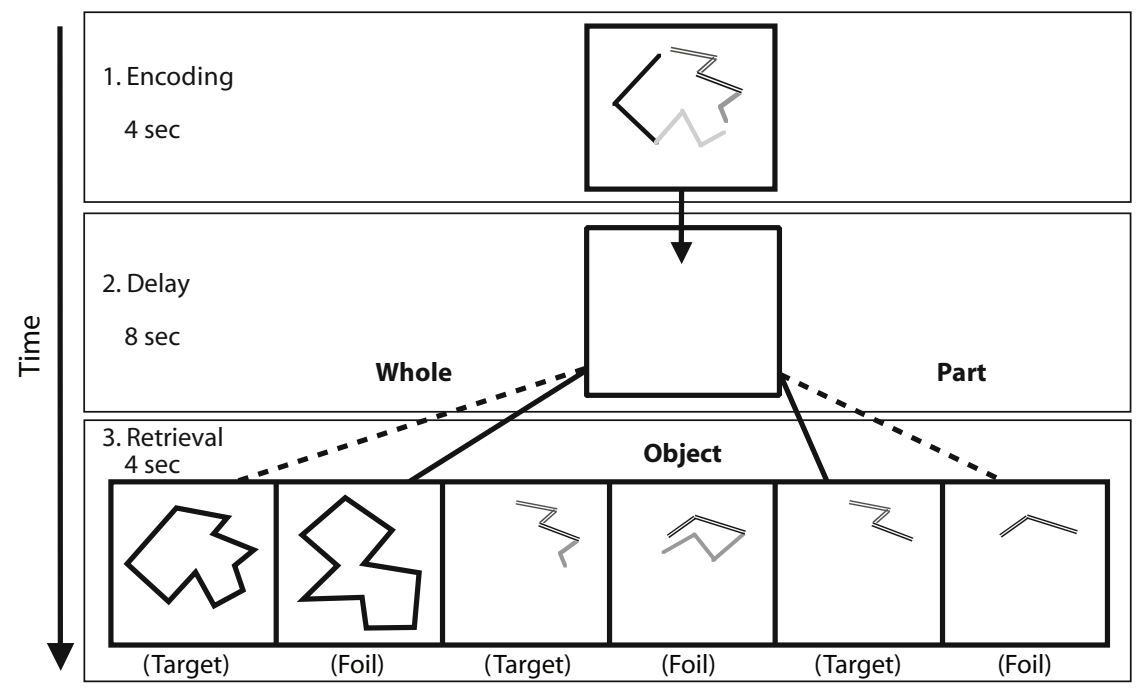

Figure 9. Stimuli used in Experiment 5. The participants in the whole, object, and part groups saw the same stimuli during the 4-sec encoding period, which was broken into two objects and four colored parts, followed by a blank screen that was presented for 8 sec. In the experiment, the lines were colored brown (solid black lines), red (light gray lines), blue (dark gray lines), and green (double lines). In the whole test condition, the participants were tested with a black 10-line-segment enclosed whole object. In the object test condition, the participants were tested with a black 5 line segment object. In the part test condition, the participants were tested with one of the 2-3 line segment colored parts shown at the beginning of the trial. An example of a target (correct) and a foil (incorrect) probe is given. 
Table 5

$A^{\prime}$ and Response Time (RT, in Milliseconds) Results in Part, Object, and Whole Groups

\begin{tabular}{|c|c|c|c|c|c|c|c|c|c|c|c|c|}
\hline \multirow[b]{3}{*}{ Type of Probe } & \multicolumn{4}{|c|}{ Part Group } & \multicolumn{4}{|c|}{ Object Group } & \multicolumn{4}{|c|}{ Whole Group } \\
\hline & \multicolumn{2}{|c|}{$A^{\prime}$} & \multicolumn{2}{|c|}{ RT } & \multicolumn{2}{|c|}{$A^{\prime}$} & \multicolumn{2}{|c|}{ RT } & \multicolumn{2}{|c|}{$A^{\prime}$} & \multicolumn{2}{|c|}{ RT } \\
\hline & $M$ & $S E$ & $M$ & $S E$ & $M$ & $S E$ & $M$ & $S E$ & $M$ & $S E$ & $M$ & $S E$ \\
\hline Part & .730 & .033 & 1,198 & 71.1 & .745 & .042 & 1,233 & 63.8 & .701 & .026 & 1,245 & 70.8 \\
\hline Object & .848 & .021 & 1,179 & 65.8 & .844 & .030 & 1,187 & 63.1 & .820 & .026 & 1,255 & 72.2 \\
\hline Whole & .897 & .030 & 1,114 & 72.5 & .926 & .020 & 1,113 & 89.9 & .904 & .024 & 1,218 & 90.8 \\
\hline
\end{tabular}

object better than parts of whole objects. Parts that were not individual objects were recalled less accurately than parts that were objects. Thus, the results support the hierarchical representation theory of visual WM. That hypothesis predicted that accuracy would be best for global configural details, followed by object-based details and then part-based details.

Instructions to focus attention on nonglobal configural visual information did not affect the global configural bias. Thus, the use of global configural information in visual WM appears to be obligatory and unaffected by top-down control.

\section{GENERAL DISCUSSION}

In this study, we conducted five experiments that examined three different levels of representation in visual WM: global configural, object based, and part based. The first four experiments contrasted WM performance for two levels of representation. In Experiment 1, we showed that accuracy for a whole object made up of 5 conjoined line segments was better than that for parts of that whole object. Note that in Experiment 1, global configural and objectbased information represented the same information, because the only object present in each stimulus was the whole object. In Experiments 2 and 3, we demonstrated that global configural information has a privileged status in visual WM, as compared with object-based information. In Experiment 4, using parts defined by color, we replicated the results of Experiments 2 and 3, indicating that the global configural bias is not limited to situations in which the parts are defined by gaps. Experiment 4 also demonstrated that instructions had no effect on how the participants responded to probes. These results suggest that the primacy of global configural information in visual WM is not subject to top-down modulation. Note that in Experiments 2-4, the role of part-based representation per se was not tested, because each part of the whole object that was tested could also be considered an object. In Experiment 5, when the three visual representation levels were compared simultaneously, accuracy was highest for global configural information, followed by object-based information and then part-based information.

These results are consistent with a hierarchical representation hypothesis that the visual WM system prioritizes global configural information, followed by object-based and, finally, part-based information. We hypothesize that the bias to store the global configural properties of a whole object in WM, instead of parts of a whole object, may reduce the total memory load, because each part would occupy critical space in a limited-capacity WM system. Whole-object representations would tax WM storage space considerably less.

The results did not support an attention-directed theory of visual WM representation. When the participants were explicitly instructed to focus on object- or part-based information, their performance did not indicate enhanced WM for this information at the expense of global configural representation. Support for the idea that the participants could not consciously control this bias came from our results showing that memory for whole objects was impervious not only to instructional demands, but also to task demands supporting memory for parts of whole objects. These results are consistent with previous results showing that the overall spatial configuration of a visual display is automatically encoded in visual WM (Jiang et al., 2004; Jiang et al., 2000; Santa, 1977). Our results extend those of past studies by showing that global configural information provides a central organizing principle in visual WM even in a task oriented toward object-based information. In essence, there is a bias to remember the global whole object, even when the parts themselves consist of objects and despite competing task-demands.

In these experiments, we endeavored to examine visual WM in isolation from visual LTM or verbal WM. The inability to use other memory systems may explain why the error rates in these studies were higher than those in other visual studies (e.g., Carlson-Radvansky, 1999; Kimchi, 2000; Olson \& Chun, 2002). Future work is necessary to determine how the hierarchical visual WM representation system affects learned visual patterns in LTM. In this enterprise, it will also be important to tease apart im-

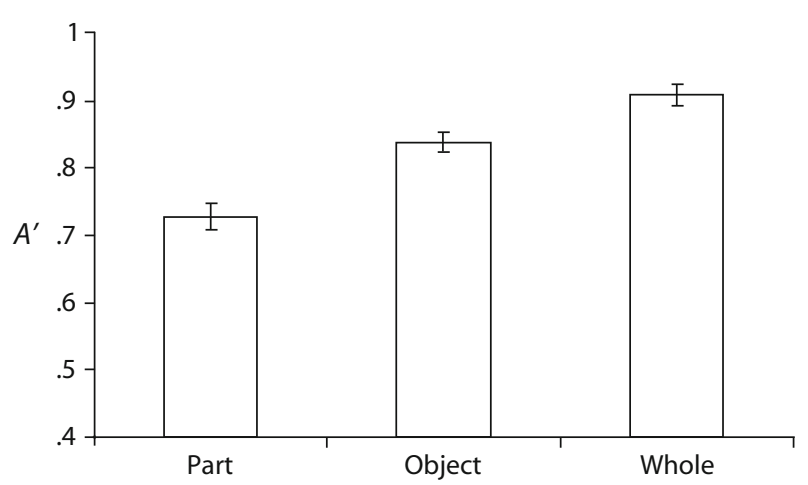

Figure 10. Effect of test condition on accuracy in Experiment 5. Whole objects were recalled significantly better than objects and parts. Objects were recalled significantly better than parts. 
plicit and explicit visual learning. Several studies have demonstrated that implicit visual learning may be used to supplement the severe capacity limits of visual WM (Song \& Jiang, 2005). Implicit visual learning is extremely sensitive to between-trial repetition. Previous research has demonstrated that implicit learning can occur in visual search tasks only if the target is in the same location in each trial (Chun \& Jiang, 1998; Olson \& Chun, 2002) and if global configural properties are not rotated between trials (Chua \& Chun, 2003). In addition, in visual search tasks, implicit learning of repeated details located in the opposite hemifield of the target does not occur if there is nonrepeating noise between the target and the repeating items (Olson, Jiang, \& Moore, 2005). However, if the entire display contracts or expands but global layout remains the same, visual learning does occur (Jiang \& Wagner, 2004; see also Carlson-Radvansky, 1999). Similar to these results, participants showed higher accuracy in recalling the outer lines of whole objects when there were no inner lines than when inner lines were present. Future work is necessary to determine the exact form of the global configural information that is represented in visual WM. It is important to note that in each experiment in this report, the global configural properties were clearly defined so that the stimuli could be grouped into a whole object. It is not clear how strong global configural effects would be when the global configural properties do not support similar grouping (see Kimchi, 2000).

In these experiments, we did not attempt to isolate the WM period during which the global configural bias occurred. It is not clear whether the effects are due to an encoding, maintenance, or retrieval bias. One approach to answer this question would be to look at interference effects of dual tasks during different WM periods (e.g., Morey \& Cowan, 2005).

Future work may also examine individual differences. Are some individuals more flexible in visual WM encoding? Vogel, McCollough, and Machizawa (2005) demonstrated that individuals who are able to ignore irrelevant visual stimuli have higher visual WM capacity than do participants who are unable to ignore irrelevant visual stimuli. In future work, we would like to examine whether some individuals have more ability to flexibly adapt the contents of visual WM. In addition, the lack of flexibility of visual WM in these experiments may be due to the long delay period during which the participants are required to hold information. We are currently examining whether participants are more flexible when they are cued to remember specific areas of visual stimuli at encoding (Sperling, 1960). Vecera, Behrmann, and McGoldrick (2000) demonstrated the attentional flexibility with which participants could focus on the part or object levels, but more work remains to determine whether there are situations in which visual WM can flexibly process information at different visual representational levels.

\section{Conclusions}

In five experiments, we demonstrated that participants had a strong bias to remember objects in terms of holistic representations, rather than as an exhaustive collection of parts. This bias appears to be impervious to direction of attention to parts. Grouping parts together as objects may reduce the difficulty of interpreting the environment, possibly by allowing rapid LTM matching, reducing overall WM load, and allowing reconstruction of visual details on the basis of long-term semantic memory for the generic properties of known objects. These results are consistent with a hierarchical theory that states that WM systems preferentially represent global configural information, followed by object-based and then part-based information. Further research is necessary to determine whether there are other situations in which object- or part-based information takes priority in visual WM over global configural information and how visual WM interacts with other memory systems (e.g., Carlson-Radvansky, 1999; Song \& Jiang, 2005).

\section{AUTHOR NOTE}

Correspondence concerning this article should be addressed to B. Rypma, School of Behavioral and Brain Sciences and Department of Psychiatry, University of Texas at Dallas and University of Texas Southwestern Medical Center, Green Hall, GR 41, Box 830688, Richardson, TX 75083-0688 (e-mail: bart.rypma@utd.edu).

\section{REFERENCES}

Baker, C. I., Olson, C. R., \& Behrmann, M. (2004). Role of attention and perceptual grouping in visual statistical learning. Psychological Science, 15, 460-466.

Behrmann, M., Zemel, R. S., \& Mozer, M. C. (1998). Object-based attention and occlusion: Evidence from normal participants and a computational model. Journal of Experimental Psychology: Human Perception \& Performance, 24, 1011-1036.

Biederman, I., Glass, A. L., \& Stacy, E. W. (1973). Searching for objects in real-world scenes. Journal of Experimental Psychology, 97, 22-27.

CARLSON-RADVANSKY, L. A. (1999). Memory for relational information across eye movements. Perception \& Psychophysics, 61, 919-934.

Carlson-Radvansky, L. A., \& Irwin, D. E. (1995). Memory for structural information across eye movements. Journal of Experimental Psychology: Learning, Memory, \& Cognition, 21, 1441-1458.

ChuA, K.-P., \& Chun, M. M. (2003). Implicit scene learning is viewpoint dependent. Perception \& Psychophysics, 65, 72-80.

Chun, M. M., \& JiAng, Y. (1998). Contextual cueing: Implicit learning and memory of visual context guides spatial attention. Cognitive Psychology, 36, 28-71.

Duff, S. C., \& Logie, R. H. (1999). Storage and processing in visuospatial working memory. Scandinavian Journal of Psychology, 40, 251-259.

Duncan, J. (1984). Selective attention and the organization of visual information. Journal of Experimental Psychology: General, 113, 501-517.

InTRAub, H. (1997). The representation of visual scenes. Trends in Cognitive Sciences, 1, 217-222.

JiAng, Y., Chun, M. M., \& Olson, I. R. (2004). Perceptual grouping in change detection. Perception \& Psychophysics, 66, 446-453.

JiAng, Y., Olson, I., \& CHUn, M. (2000). Organization of visual shortterm memory. Journal of Experimental Psychology: Learning, Memory, \& Cognition, 26, 683-702.

JiANG, Y., \& WAGNER, L. C. (2004). What is learned in spatial contextual cuing - configuration or individual locations? Perception \& Psychophysics, 66, 454-463.

KImcHI, R. (2000). The perceptual organization of visual objects: A microgenetic analysis. Vision Research, 40, 1333-1347.

Kumar, A., \& JiAnG, Y. (2005). Visual short-term memory for sequential arrays. Memory \& Cognition, 33, 488-498.

Logie, R. H. (1995). Visuo-spatial working memory. Hove, U.K.: Erlbaum. 
LucK, S. J., \& Vogel, E. K. (1997). The capacity of visual working memory for features and conjunctions. Nature, 390, 279-281.

Morey, C. C., \& Cowan, N. (2005). When do visual and verbal memories conflict? The importance of working-memory load and retrieval. Journal of Experimental Psychology: Learning, Memory, \& Cognition, 31, 703-713.

Olson, I. R., \& Chun, M. M. (2002). Perceptual constraints on implicit learning of spatial context. Visual Cognition, 9, 273-302.

Olson, I. R., JiAng, Y., \& Moore, K. S. (2005). Associative learning improves visual working memory performance. Journal of Experimental Psychology: Human Perception \& Performance, 31, 889-900.

PALMER, S. E. (1977). Hierarchical structure in perceptual representation. Cognitive Psychology, 9, 441-474.

Palmer, S. E., \& RocK, I. (1994). Rethinking perceptual organization: The role of uniform connectedness. Psychonomic Bulletin \& Review, 1, 29-55.

Pollack, I., \& Norman, D. A. (1964). A non-parametric analysis of recognition experiments. Psychonomic Science, 1, 125-126.

Rock, I., \& GuTMAN, D. (1981). The effect of inattention on form perception. Journal of Experimental Psychology: Human Perception \& Performance, 7, 275-285.

RYPMA, B. (2006). Factors controlling neural activity during delayedresponse task performance: Testing a memory organization hypothesis of prefrontal function. Neuroscience, 139, 223-235.

Rypma, B., Berger, J. S., \& D'Esposito, M. (2002). The influence of working-memory demand and subject performance on prefrontal cortical activity. Journal of Cognitive Neuroscience, 14, 721-773.

SANTA, J. L. (1977). Spatial transformations of words and pictures. Journal of Experimental Psychology: Human Learning \& Memory, 3, 418-427.

Schneider, W., Eschman, A., \& Zuccolotto, A. (2002). E-prime user's guide. Pittsburgh: Psychology Software Tools.

Simons, D., \& Chabris, C. (1999). Gorillas in our midst: Sustained inattentional blindness for dynamic events. Perception, 28, 1059-1074.

SonG, J., \& JIANG, Y. (2005). Connecting the past with the present: How do humans match an incoming visual display with visual memory. Journal of Vision, 5, 322-333.

SPERLING, G. (1960). The information available in brief visual presentations. Psychological Monographs: General \& Applied, 74, 1-29.

SPERLING, G. (1967). Successive approximations to a model of short term memory. Acta Psychologica, 27, 285-292.
Vecera, S. P., Behrmann, M., \& Filapek, J. C. (2001). Attending to the parts of a single object: Part-based selection limitations. Perception \& Psychophysics, 63, 308-321.

Vecera, S. P., Behrmann, M., \& McGoldrick, J. (2000). Selective attention to the parts of an object. Psychonomic Bulletin \& Review, 7, 301-308.

Vogel, E. K., McCollough, A. W., \& Machizawa, M. G. (2005). Neural measures reveal individual differences in controlling access to working memory. Nature, 438, 500-503.

Vogel, E. K., Woodman, G. F., \& LuCK, S. J. (2001). Storage of features, conjunctions and objects in visual working memory. Journal of Experimental Psychology: Human Perception \& Performance, 27, 92-114.

Woodman, G. F., Vecera, S. P., \& LucK, S. J. (2003). Perceptual organization influences visual working memory. Psychonomic Bulletin \& Review, 10, 80-87.

\section{NOTES}

1. Long-term visual memory performance may also rely upon object-based attention. In one study, in which participants were shown two shapes at one time but were instructed to attend to only one of the shapes, the participants more accurately recalled the unattended shapes when they were connected to the attended shape by a line than when the shapes were not connected (Baker, Olson, \& Behrmann, 2004). Rock and Gutman (1981) demonstrated that when two overlapping outlines of novel shapes of different colors were presented and participants were told to make a pleasantness judgment on only one of them, memory was very low for the unattended object. Simons and Chabris (1999) showed that participants instructed to watch a ball game can fail to notice objects as distinctive as a person in a gorilla suit, even when the object conspicuously traverses the participants' visual field.

2. $A^{\prime}$ was calculated by the following formula: If fa(false alarms) $>$ hit: $A^{\prime}=[(\mathrm{fa}-$ hit $) *(1+\mathrm{fa}-$ hit $)] /[4 * \mathrm{fa} *(1-$ hit $)]$. If hit $>$ fa: $A^{\prime}=.5+$ $[($ hit - fa $) *(1+$ hit - fa $)] /[4 *$ hit $*(1-$ fa $)]$.

3 . We would like to thank an anonymous reviewer for suggesting this alternative explanation.

(Manuscript received March 4, 2005; revision accepted for publication April 7, 2006.) 